\title{
DIAGNOSTIC ACCURACY OF ELECTROCARDIOGRAPHY (ECG) FOR THE DIAGNOSIS OF LEFT VENTRICULAR HYPERTROPHY, TAKING ECHOCARDIOGRAPHY AS GOLD STANDARD
}

\author{
Muhammad Fahad, Ayesha Nawaz, Khalid Mehmood, Rameesha Hussain, Raza Ahmad \\ Department of Medical Unit 1, Services Hospital, Lahore - Pakistan
}

\begin{abstract}
Objective: To determine the diagnostic accuracy of Electrocardiography (ECG) of Left Ventricular Hypertrophy, taking echocardiography as a gold standard.

Mterial \& Methods: It was a Cross-Sectional Study. The study was conducted in the Medicine Department, Services Hospital, Lahore from February 19, 2018, to August 19, 2018. A total of 300 cases were enrolled. Standardized 12-lead ECG was used in subjects by Cardiofax electrocardiograph paper speed at $25 \mathrm{~mm} / \mathrm{sec}$. Two dimensionally (2D) guided M Mode echocardiographic measurements were taken and the presence/absence of LVH was recorded. The collected data was entered and analyzed in computer software SPSS software v25.0. A 2x2 table was drawn to calculate the sensitivity, specificity, positive predictive value, negative predictive value and diagnostic accuracy of ECG for LVH taking Echocardiographic findings as a gold standard.
\end{abstract}

Results: Out of 300 cases, $172(57.33 \%)$ were male and $128(42.67 \%)$ as females. The mean age was $38.72 \pm 5.64$ years. Accuracy of electrocardiography (ECG) for LVH diagnosis taking echocardiography as the gold standard was calculated as $85.21 \%, 84.18 \%, 82.88 \%, 86.36 \%$ and $84.67 \%$ as Sn, Sp, PPV, NPV, and diagnostic accuracy respectively.

Conclusion: Diagnostic accuracy of Electrocardiography (ECG) for LVH diagnosis is good and this diagnostic modality can be used in areas where echocardiography is not available.

Keywords: Electrocardiography (ECG), Diagnostic Accuracy, Echocardiography, Left Ventricular Hypertrophy..

This article may be cited as: Fahad M, Nawaz A, Mehmood K, Hussain R, Ahmad R. Diagnostic accuracy of electrocardiography (ECG) for the diagnosis of left ventricular hypertrophy, taking echocardiography as gold standard. J Med Sci 2021 April;29(2):66-69

\section{INTRODUCTION}

Left ventricular hypertrophy (LVH) is an independent, solid indicator of cardiovascular mortality and morbidity $^{1}$. It is currently valued that $\mathrm{LVH}$ is intervened by different neuro-hormonal substances that autonomously apply trophic consequences for myocytes and non-myocytes in the heart ${ }^{2}$. Choices about treatment ought to be founded on appraisals of hypertensive target organ harm and in general cardiovascular hazards. Different ECG criteria have been advocated, however, there is little data with regards to the prescient estimations of the particular criteria for the right determination. Above all, the clinical utility of ECG has been restricted by its inability to study the structures in details ${ }^{3}$. Echocardiography is a typical way to deal with survey the myocardial structures, which

\footnotetext{
Correspondence

Dr. Muhammad Fahad

Department of Medical Unit 1, Services Hospital,

Lahore - Pakistan

Email: muhammad_fahad_666@yahoo.com

Cell: +92-337-7116565
}

Date received: $21-12-2020$

Date revised: $\quad 30-01-2021$

Date accepted: $04-05-2021$ can give a quantitative assessment of LV bulk and the qualities are close to those found at necropsy ${ }^{4,5}$. Echocardiography (echo) is exact yet also increasingly costly system.

The introduction of the practice of ECG in diagnosing left ventricular hypertrophy has proven its benefits when compared to $\mathrm{ECHO}$ as the gold standard for the validation of ECG as a tool to diagnose LVH would benefit centers that lack access to echocardiography. Moreover, in developing countries, echocardiography can't be prescribed to screen each patient with hypertension, starting assessment utilizing ECG can help in choosing the individuals who require echocardiography ${ }^{6,7}$. In an Indian study, the sensitivity of ECG was $43.5 \%$ and specificity was 88.9 when it was used for diagnosis of LVH keeping $\mathrm{ECHO}$ as a gold standard, ${ }^{8}$ while another recent study recorded these findings as to the sensitivity of $86 \%$ and specificity of $81 \%$ 9 . A higher hazard of cardiac morbidity and mortality, left ventricular hypertrophy is linked, however, its earlier exposure is important especially among those patients who are having hypertension or other associated cardiovascular etiologies. ECG for detection of LVH is used as the common diagnostic tool but the findings of different studies showing different results. However, we planned this study 
Diagnostic Accuracy Of Electrocardiography (Ecg) For The Diagnosis Of Left Ventricular Hypertrophy, Taking....

to determine the diagnostic accuracy of Electrocardiography (ECG) for the diagnosis of $\mathrm{LVH}$ taking echocardiography as a gold standard. As no local data is available to evaluate electrocardiography in the diagnosis of left ventricular hypertrophy and variability is also seen in the sensitivity and specificity of previously conducted studies. This research will be beneficial in making the diagnosis of left ventricular hypertrophy cost-effective and with high accuracy comparable to echocardiography without exposing the patient to harmful ionizing radiation.

\section{MATERIAL \& METHODS}

It was a Cross-Sectional Study. The study was conducted in the Medicine Department, Services Hospital, Lahore from February 19, 2018, to August 19, 2018. A total of 300 patients fulfilling inclusion criteria (patients of both genders having ages between 20-50 years, patients with clinically diagnosed LVH with the displaced apex beat and with well-sustained heave) were included. The exclusion criteria were; patients using drugs or on digital therapy, that could amend ECG (on history and medical record) and patients with ischemic heart disease and lung disease due to obstruction (on history and medical record). The sample size of 300 cases was calculated by using a $95 \%$ confidence level with an expected percentage of sensitivity as $43.5 \%$ with a $7 \%$ margin of error, specificity $88 \%$ with $8 \%$ margin of error 9 with an expected percentage of LVH in $84 \% 11$ of patients of hypertension. Standardized a 12-lead ECG was used in subjects by Cardiofax electrocardiograph paper speed at $25 \mathrm{~mm} / \mathrm{sec}$. and with an amplitude of $1 \mathrm{mV} / \mathrm{cm}$ of stylus deflection. Findings of $\mathrm{LVH}$ (according to operational definition) on ECG were recorded as the presence/absence of LVH by the researcher herself. Then echocardiography of all patients was done by a well-trained echocardiographer, using Toshiba Applio 50 echocardiography system using $2.5 \mathrm{MHz}$ transducer. According to the American Society of Echocardiography, two-dimensionally (2D) guided M Mode echocardiographic measurements were taken and the presence/absence of $\mathrm{LVH}$ was recorded.

LVH assessed on Echocardiography: Those patients whose echocardiography revealed concentric LVH and LVM1 $\geq 116 \mathrm{~g} / \mathrm{m} 2$ and $104 \mathrm{~g} / \mathrm{m} 2$ for females. Left ventricular mass thickening was calculated by the following formula: LV mass $=0.8 \times 1.04$ [(IVS + LVID + LVPW) 3 $-($ LVID)3] + 0.6 LV mass Index = LVM1: LV mass (g)/ BSA (m2) LVH on Electrocardiography (ECG): Twelve lead ECG was taken adopting the standard procedure and LVH was diagnosed based on the voltage criteria: $\mathrm{S}$ in (V1 or V2) + R in (V5 or V6 $\geq 35 \mathrm{~mm}$ ).

The collected data was entered and analyzed in computer software SPSS v25.0. The quantitative data like age was presented as Mean \pm S.D. Qualitative variables like gender and presence/absence of LVH on ECG \& echocardiography were presented as frequency and per- centages. A 2x2 table was drawn to calculate the sensitivity, specificity, positive predictive value, negative predictive value and diagnostic accuracy of ECG for LVH taking Echocardiographic findings as a gold standard.

\section{RESULTS}

A total of 300 cases fulfilling the inclusion criteria were included to assess the diagnostic accuracy of Electrocardiography (ECG) for the diagnosis of Left Ventricular Hypertrophy taking echocardiography as gold-standard. According to the age distribution, it was noted that $83(27.67 \%)$ were between $20-35$ years of age, while $217(72.33 \%)$ were between $36-50$ years of age. The mean age was as $38.72 \pm 5.64$ years.

Gender distribution of the patients was done which showed that $172(57.33 \%)$ were male and $128(42.67 \%)$ as females. The frequency of LVH on echocardiography showed that $142(47.33 \%)$, while $158(52.67 \%)$ had no findings of LVH. Accuracy of electrocardiography (ECG) for LVH diagnosis taking echocardiography as the gold standard was calculated as $85.21 \%, 84.18 \%, 82.88 \%, 86.36 \%$ and $84.67 \%$ as Sn, Sp, PPV, NPV, and diagnostic accuracy

Table 1: Frequency distribution of LVH on Electrocardiography

\begin{tabular}{|c|c|c|}
\hline LVH on Electrocardiography & Frequency & Percent \\
\hline Yes & 146 & 48.67 \\
\hline No & 154 & 51.33 \\
\hline Total & 300 & 100.0 \\
\hline
\end{tabular}

Table 2: Frequency distribution of LVH on Electrocardiography

\begin{tabular}{|c|c|c|}
\hline LVH on Electrocardiography & Frequency & Percent \\
\hline Yes & 146 & 48.67 \\
\hline No & 154 & 51.33 \\
\hline Total & 300 & 100.0 \\
\hline
\end{tabular}

Table 3: Findings of Echocardiography and Electrocardiography

\begin{tabular}{|c|c|c|c|}
\hline \multirow{2}{*}{$\begin{array}{c}\text { LVH on } \\
\text { Electrocardiography }\end{array}$} & \multicolumn{2}{|c|}{ LVH on Echocardiography } & \multirow{2}{*}{ Total } \\
\cline { 2 - 3 } & Yes & No & \\
\hline Yes & 121 & 25 & 146 \\
\hline No & 21 & 133 & 154 \\
\hline Total & 142 & 158 & 300 \\
\hline
\end{tabular}

Table 4: Diagnostic accuracy of Electrocardiography.

\begin{tabular}{|c|c|}
\hline Sensitivity & $\mathbf{8 5 . 2} \%$ \\
\hline Specificity & $84.1 \%$ \\
\hline Positive predictive value & $82.8 \%$ \\
\hline Negative predictive value & $86.3 \%$ \\
\hline Accuracy & $84.6 \%$ \\
\hline
\end{tabular}


respectively.

\section{DISCUSSION}

A higher hazard of cardiac morbidity and mortality, left ventricular hypertrophy is linked, however, its earlier exposure is important especially among those patients who are having hypertension or other associated cardiovascular etiologies. ECG for detection of LVH is used as the common diagnostic tool but the findings of different studies showing different results. However, we planned this study to determine the diagnostic accuracy of Electrocardiography (ECG) for the diagnosis of LVH taking echocardiography as a gold standard.

Gender distribution of the patients was done which showed that $172(57.33 \%)$ were male and $128(42.67 \%)$ as females. The frequency of LVH on gold standard showed that $142(47.33 \%)$, while $158(52.67 \%)$ had no findings of LVH. Accuracy of electrocardiography (ECG) for LVH diagnosis taking echocardiography as the gold standard was calculated as $85.21 \%, 84.18 \%, 82.88 \%, 86.36 \%$ and $84.67 \%$ as Sn, Sp, PPV, NPV, and diagnostic accuracy respectively.

An Indian study recorded the sensitivity of ECG as $43.5 \%$ and specificity was 88.9 when it was used for diagnosis of LVH keeping ECO as a gold standard, ${ }^{9}$ our results are in contrast with this study. Another recent study recorded these findings as to the sensitivity of $86 \%$ and specificity of $81 \%$ which shows a big variation in sensitivity of ECG and needs another study to be conducted to find out its diagnostic accuracy, these findings agree with our study $^{9}$. Waqas Hameed and others, ${ }^{10} \mathrm{Sn}$ and Sp of electrocardiography (ECG) for LVH diagnosis taking echocardiography as the gold standard were calculated as 35\% and $90 \%$, our findings agree regarding the specificity of the ECG while sensitivity is higher in our study.

In another study done by Okin, et al., ${ }^{11} \mathrm{Sn}$ and Sp of electrocardiography (ECG) for LVH diagnosis taking echocardiography as the gold standard was calculated as $12 \%$ and as $100 \%$. In another study by Devereux, et al., ${ }^{12} \mathrm{Sn}$ and Sp of electrocardiography (ECG) for LVH diagnosis taking echocardiography as a gold standard was calculated as $34 \%$ and as $98 \%$. Ahmad Hasan and colleagues ${ }^{13}$ conducted a study to find out how much we can solely rely on the electrocardiography for the diagnosis of LVH and recorded that Left ventricular hypertrophy on electrocardiography, 96(48\%) patients were found positive and 104(52\%) were found negative. They concluded that the frequency of true positive cases of Left Ventricular
Hypertrophy on Electrocardiography taking Echocardiography as the gold standard is acceptable.

We are of the view that left ventricular mass determination on echocardiography is the most sensitive modality to diagnose LVH but ECG also remains a useful initial investigation and it can be used in those areas where the facility of echo is not available especially in rural areas and non-specialized centers. In this study, there are some limitations as well. First, there is a small sample size and second, this was a single centered study.

\section{CONCLUSION}

Diagnostic accuracy of Electrocardiography (ECG) for LVH diagnosis is higher and this diagnostic modality can be used in areas where echocardiography is not available.

\section{REFERENCES}

1. Seubsung A, Thirawut V, Prasertkulchai W, Tangcharoen T. P3460 Accuracy of novel EKG criteria for left ventricular hypertrophy diagnosis in elderly Thai patients using cardiovascular magnetic resonance as a gold standard. European Heart Journal. 2018;39(1):563-9.

2. Erküner Ö, Dudink EA, Nieuwlaat R, Rienstra M, Van Gelder IC, Camm AJ, et al. Effect of systemic hypertension with versus without left ventricular hypertrophy on the progression of atrial fibrillation (from the Euro Heart Survey). Am J Cardiol. 2018;122(4):578-83.

3. Mavrogeni S, Katsi V, Vartela V, Noutsias M, Markousis-Mavrogenis G, Kolovou G, et al. The emerging role of cardiovascular magnetic resonance in the evaluation of hypertensive heart disease. BMC Cardiovasc Dis. 2017;17(1):132-9.

4. Singh A, Baruah B, Baruah C. Electrocardiographic and echocardiographic evaluation of left ventricular hypertrophy in the hypertensive patients-a hospital-based study. J Evol Med Dental Sci 2018;7(10):1189-93.

5. Pinto J, George P, Hedge N. Study in Southern India among hypertensive patients using ECG to screen left ventricular hypertrophy - can we do it in rural health centers? Community Medical Section 2014;8186(1):410715.

6. Paun N, Nanea IT, Nicolae CA, Munteanu A, Plesa FC, Diaconu $\mathrm{M}$, et al. The significance of ventricular interdependence in patients with right ventricular hypertrophy and normal left ventricular function. Revista De Chimie. 2019;70(10):3689-93.

7. Venugopal K, Gadwalkar SR, Ramamurthy P. Electrocardiogram and echocardiographic study of left ventricular hypertrophy in patients with essential hypertension in a teaching medical college. J Sci Society. 2016;43(2):75. 
Diagnostic Accuracy Of Electrocardiography (Ecg) For The Diagnosis Of Left Ventricular Hypertrophy, Taking....

8. Pinto J, George P, Hegde N. Study in southern India among hypertensive patients using ECG to screen left ventricular hypertrophy-Can we do it in the rural health center. J Clin Diagnose Res 2014;89(3):59-62.

9. Michael A. bauml. Left ventricular hypertrophy: An overlooked cardiovascular risk factor. Cleveland Clin J Med. 2010;776:381-7.

10. Hameed W, Razi MS, Khan MA, Hussain MM, Aziz S, Habib S. Electrocardiographic diagnosis of left ventricular hypertrophy: comparison with echocardiography. Pak J Physiol 2005;1(1-2).

11. Okin PM, Roman MJ, Devereux RB. Electrocardiographic diagnosis of left ventricular hypertrophy by the time - voltage integral of the QRS complex. J Am Coll Cardiol 1994;23:133-40.

12. Devereux RB, Casale PN, Eisenberg RR. Electrocardiographic detection of left ventricular hypertrophy using echocardiographic determination of left ventricular mass as the reference standard. comparison of standard criteria, computer diagnosis, and physician interpretation. J Am Coll Cardiol 1984;3:82-7.

13. Hasan A, Ghous Z, Shahzad ST, Sajjad U, Tariq Z, Akram Z. Frequency of True Positive Cases of Left Ventricular Hypertrophy on Electrocardiography Taking Echocardiography as Gold Standard. JFJMC. 2014;8:62-7.
CONFLICT OF INTEREST: Authors declare no conflict of interest

GRANT SUPPORT AND FINANCIAL DISCLOSURE: NIL

\section{AUTHOR'S CONTRIBUTION}

Following authors have made substantial contributions to the manuscript as under

Fahad M: Concept, study design, discussion, manuscript writing, facilitation of the reagent and materials, critical review

Nawaz A: $\quad$ Facilitation of the reagent and materials, critical review, interpretation.

Mehmood K: Analysis, interpretation, manuscript writing, study conduction.

Hussain R: Analysis, interpretation , manuscript writing, study conduction

Ahmad R: Critical review, study conduction.

Authors agree to be accountable for all aspects of the work in ensuring that questions related to the accuracy or integrity of any part of the work are appropriately investigated and resolved. 